\title{
DESEMPENHO DE TILÁPIA DO NILO (OREOCHROMIS NILOTICUS) SUPLEMENTADA COM VITAMINA C
}

\author{
PERFORMANCE OF TILAPIA (OREOCHROMIS NILOTICUS) \\ SUPPLEMENTED WITH VITAMINC
}

\author{
Navarro, R.D. ${ }^{1 *}$, Ferreira, W.M. ${ }^{2}$, Ribeiro Filho, O.P. ${ }^{3}$, Botion, L.M. ${ }^{4}$, Pereira, F.K.S. ${ }^{5}$, \\ Silva, R.F. ${ }^{4}$ e Maciel, T.E.F. ${ }^{3}$
}

${ }^{1}$ Faculdade de Agronomia e Veterinária da Universidade de Brasília. Campus Universitário Darcy Ribeiro. Asa Norte. Brasília, DF. Brasil. rddnavarro@yahoo.com.br ${ }^{2}$ Departamento de Zootecnia. Escola de veterinária. Avda. Antônio Carlos, 6627. Caixa Postal 567. Campus da Universidade Federal de Minas Gerais. Belo Horizonte-MG. CP 30123-970. Brasil. ${ }^{3}$ Departamento de Biologia Animal. Universidade Federal de Viçosa. Brasil. ${ }^{4}$ Departamento de Fisiologia e Biofísica. Universidade Federal de Minas Gerais. Brasil. ${ }^{5}$ Universidade Federal de Lavras. Lavras Minas Gerais. Brasil.

\section{PalaVRas chave adicionais}

Exigência nutricional. Ácido ascórbico. Nutrição de peixes.

\section{RESUMO}

Objetivou-se avaliar a suplementação de vitamina $\mathrm{C}$ no desempenho produtivo de tilápias revertidas (Oreochromis niloticus). Foram utilizadas 400 pós-larvas revertidas num delineamento inteiramente ao acaso com cinco tratamentos $(0$, $50,100,150$ e $200 \mathrm{mg} / \mathrm{kg}$ de vitamina C monofosfato de ácido ascórbico $L$ ) numa ração isoprotéica de $36 \%$ de PB e isocalórica $3600 \mathrm{kcal}$ de ED $/ \mathrm{kg}$ com 4 repetições. Observou-se efeito significativo no peso médio final e ganho de peso com 50, 100 e $200 \mathrm{mg}$ de vitamina C por kg. Não foi observada diferença significativa para o comprimento total. No entanto, para o comprimento padrão foi observada diferença significativa com os tratamentos 50,100 e $200 \mathrm{mg} / \mathrm{kg}$. Verificou-se efeito significativo na taxa de eficiência protéica com suplementação de 50, 100, 150 e $200 \mathrm{mg} / \mathrm{kg}$ de vitamina C. A suplementação de vitamina $C$ não influenciou a porcentagem de MS, proteína bruta e porcentagem de proteína no ganho de peso. Não foi observada diferença significativa dos tratamentos para extrato etéreo, porcentagem de gordura no ganho de peso. A suplementação de vitamina $C$ não influenciou significativamente a concentração de glicogênio no músculo. Não foi observada diferença significativa para glicogênio do fígado.

Recibido: 17-11-08. Aceptado: 5-3-09.

\section{Additional KeYWORDS}

Nutritional requirement. Ascorbic acid. Nutrition of fish.

\section{SUMMARY}

The objective of this study was to evaluate the supplementation of Vitamin $\mathrm{C}$ in the productive performance of reverted tilapias (Oreochromis niloticus). Four hundred tilapias were used. The experiment was mounted according to a completely randomized design with 5 treatments $(0,50,100$, 150 and $200 \mathrm{mg} / \mathrm{kg}$ of vitamin C monophosphate of ascorbic acid $\mathrm{L}$ ) in a ration isoproteinic ( $36 \%$ of $\mathrm{PB}$ ) and isocaloric (3600 kcal of DE/ $\mathrm{kg}$ ) with 4 repetitions. Significant effect was observed in final weight and weight gain for in treatments of 50,100 and $200 \mathrm{mg} / \mathrm{kg}$ of vitamin C. It was not observed significant difference for the total length. However, for the standard length was observed significant difference in treatments 50, 100 and $200 \mathrm{mg} / \mathrm{kg}$ of vitamin C. Significant effect was observed for protein efficiency rate with supplementation of $50,100,150$ and $200 \mathrm{mg} / \mathrm{kg}$ of vitamin C. Vitamin C supply did not influenced MS percentage, crude protein and protein percentage in weight gain. Significant difference was not observed for ether extract and fat percentage in weight gain. Vitamin $C$ supply did not influence significantly the concentration of muscle glycogen. It was not observed significant difference for liver glycogen.

Arch. Zootec. 59 (228): 589-596. 2010. 
NAVARRO, FERREIRA, RIBEIRO FILHO, BOTION, PEREIRA, SILVAE MACIEL

\section{INTRODUÇÃO}

A tilápia Oreochromis niloticus, é considerada o peixe mais importante do século XXI, cultivado em mais de 100 países e também no Brasil devido sua rusticidade e rápido crescimento, sendo a sua carne considerada de ótima qualidade (Kubitza, 2000). O interesse pelo cultivo dessa espécie, no Sul e Sudoeste do país, cresceu rapidamente nos últimos oito anos em virtude da tecnologia de reversão sexual e da pesca esportiva representada pelos pesque-pagues. A tilápia é criada em diversos sistemas, desde a cultura semi-intensiva em tanques que recebem dejetos animais, como em cultivos intensivos em raceways e tanquesrede (Kubitza, 2000).

O desenvolvimento eficiente e saudável dos animais passa obrigatoriamente pelo fornecimento de uma dieta que satisfaz as necessidades básicas de crescimento, contendo concentrações próximas do ideal e seus diversos componentes, aliados a tecnologia de preparação. A estocagem, a concentração de vitaminas e minerais, a biodisponibilidade dos nutrientes são exemplos de parâmetros que interferem no desenvolvimento do animal (Navarro et al., 2007).

Entre os micronutrientes, a vitamina Cé essencial para a maioria das espécies de peixes que necessitam desse nutriente na dieta, por não a sintetizarem, devido à ausência da enzima gulonolactona oxidase que transforma glucose em ácido ascórbico (Merchie et al., 1997 e Rotta, 2003). A vitamina $\mathrm{C}$ atua no organismo como co-fator para diversas reações, entre elas: a hidroxilação da prolina na síntese de colágeno (Matty, 1985; Lee e Bai, 1998 e Rotta, 2003).

Uma das formas de armazenamento de energia consumida como alimentos pelo peixe é o glicogênio. O glicogênio é encontrado em grande quantidade nos tecidos do fígado e do músculo dos peixes. Embora o tecido muscular de peixes carnívoros, como a truta arco íris, possa concentrar cerca de $6 \%$ a mais de glicogênio que o fígado, as quantidades totais de glicogênio muscular ou hepático podem ser consideradas iguais (Cyrino et al., 2000, Navarro et al., 2006).

Além disso, poucos trabalhos têm feito às exigências nutricionais e desempenho produtivo com reservas energéticas corporais (Cyrino et al., 2000). Nesse sentido, objetivou-se avaliar a suplementação de vitamina $\mathrm{C}$ no desempenho de tilápias revertidas (Oreochromis niloticus).

\section{MATERIALE MÉTODOS}

O experimento foi conduzido no ranário experimental do Departamento e Biologia Animal - UFV - no período de 23/01/2005 a 23/03/2005.

Foram utilizados 400 tilápias revertidas (Oreochromis niloticus) com peso e comprimento inicial de $1,88 \pm 0,88$ g e $8,39 \pm$ $0,40 \mathrm{~cm}$ respectivamente. As pós-larvas foram distribuídas em 20 aquários com capacidade de 10001 renovação de água constante $7,5 \mathrm{ml} /$ minuto $\mathrm{O}$ experimento foi montado segundo um delineamento feito inteiramente ao acaso com cinco tratamentos de suplementação de vitamina $\mathrm{C}(0,50,100$, 150 e $200 \mathrm{mg} / \mathrm{kg}$ de monofosfato de ácido ascórbico L) em uma ração isoproteíca de $36 \%$ de $\mathrm{PB}$ e isocalórica $3600 \mathrm{kcal}$ de $\mathrm{ED} / \mathrm{kg}$ com quatro repetições (tabela I). A fase de adaptação foi de cinco dias.

As dietas experimentais foram peletizadas, secas em estufa de circulação forçada a $55^{\circ} \mathrm{C}$ e, posteriormente, fracionadas nos diâmetros de $1 \mathrm{~mm}$, entre $4 \mathrm{~mm}$ a $6 \mathrm{~mm}$, sendo utilizadas de acordo com o tamanho dos peixes. As dietas permaneceram armazenadas a $-20^{\circ} \mathrm{C}$ até sua utilização. As dietas experimentais foram peletizadas e a oferta de ração foi de $5 \%$ do peso vivo, sendo ajustada a cada 15 dias. Foram realizadas despescas com rede de malha de $3 \mathrm{~cm}$ entre nós, sendo capturado $15 \%$ dos animais. As biometrias foram realizadas com auxílio de paquímetro e balança de precisão. Os peixes 


\section{DESEMPENHO DE TILÁPIA DO NILO COM VITAMINAC}

foram alimentados três vezes ao dia (8:00, 13:00 e 18:00 horas). Os aquários foram sifonados, diariamente, para retirar sobras de ração e fezes. O fotoperíodo foi de 12 horas. A medição da temperatura da água foi realizada diariamente, às 7:00 e às 17:00, enquanto o $\mathrm{pH}$ e o oxigênio dissolvido foram medidos a cada sete dias.

No final do experimento, após jejum de
24 horas, os peixes foram abatidos por insensibilização (caixas isotérmicas com gelo moído e água na proporção $1: 1$ ) de acordo com Navarro et al. (2007). Depois do abate, foram eviscerados. Para realizar as análises bromatológicas, as carcaças foram secas em estufa com ventilação forçada a $55^{\circ} \mathrm{C}$ por $48 \mathrm{~h}$. Após a secagem, passaram por uma moagem em moinho de bola, até

Tabela I. Composições percentuais, químicas e calculadas das dietas experimentais. (Chemical composition of experimental diets as fed).

\begin{tabular}{|c|c|c|c|c|c|}
\hline & \multicolumn{5}{|c|}{ Dietas (vitamina C mg/kg) } \\
\hline & 0 & 50 & 100 & 150 & 200 \\
\hline \multicolumn{6}{|l|}{ Ingredientes (\%) } \\
\hline Farelo de soja & 21,50 & 21,50 & 21,50 & 21,50 & 21,50 \\
\hline Glúten de milho & 30,00 & 30,00 & 30,00 & 30,00 & 30,00 \\
\hline Milho & 28,50 & 28,50 & 28,50 & 28,50 & 28,50 \\
\hline Farinha de peixe & 9,00 & 9,00 & 9,00 & 9,00 & 9,00 \\
\hline Oleo de soja & 7,60 & 7,60 & 7,60 & 7,60 & 7,60 \\
\hline Fosfato bicalcio & 1,37 & 1,37 & 1,37 & 1,37 & 1,37 \\
\hline Calcário & 0,51 & 0,51 & 0,51 & 0,51 & 0,51 \\
\hline Sal & 0,40 & 0,40 & 0,40 & 0,40 & 0,40 \\
\hline Premix isento de ácidoL-ascórbico ${ }^{3}$ & 0,60 & 0,60 & 0,60 & 0,60 & 0,60 \\
\hline Metionina & 0,34 & 0,34 & 0,34 & 0,34 & 0,34 \\
\hline Lisina & 0,15 & 0,15 & 0,15 & 0,15 & 0,15 \\
\hline BHT (antioxidante) & 0,02 & 0,02 & 0,02 & 0,02 & 0,02 \\
\hline Vitamina C mg/kg & 0 & 50 & 100 & 150 & 200 \\
\hline \multicolumn{6}{|l|}{ Níveis nutricionais } \\
\hline Proteína bruta $\% 1$ & 36 & 36 & 36 & 36 & 36 \\
\hline Energia digestível ${ }^{2}, \mathrm{kcal} / \mathrm{kg}$ & 3600 & 3600 & 3600 & 3600 & 3600 \\
\hline Fibra bruta \% & 2,30 & 2,30 & 2,30 & 2,30 & 2,30 \\
\hline Lisina \% & 1,50 & 1,50 & 1,50 & 1,50 & 1,500 \\
\hline Metionina + cistina \% & 1,11 & 1,11 & 1,11 & 1,11 & 1,11 \\
\hline Treonina \% & 1,35 & 1,35 & 1,35 & 1,35 & 1,35 \\
\hline Triptófano $\%{ }^{2}$ & 0,29 & 0,29 & 0,29 & 0,29 & 0,29 \\
\hline Cálcio \% & 1,04 & 1,04 & 1,04 & 1,04 & 1,04 \\
\hline Fósforo total $\%{ }^{2}$ & 0,80 & 0,80 & 0,80 & 0,80 & 0,80 \\
\hline Relação ED: PB & 10 & 10 & 10 & 10 & 10 \\
\hline
\end{tabular}

${ }^{1}$ Com base nas análises de laboratório LNA/DZO.

${ }^{2}$ Baseados nos valores propostos pelo NRC (1993) e por Rostagno (2000).

${ }^{3}$ Premix comercial $(5 \mathrm{~kg} / \mathrm{t})$, com níveis de garantia por quilograma de produto: Vit. A, $1200000 \mathrm{UI}$; Vit. $\mathrm{D}_{3}$, $200000 \mathrm{Ul}$; Vit k, 2400 mg; Vit $B_{3} .4800$ mg; Vit $B_{2} 4800$ mg, Vit B $B_{6}, 4000$ mg, Vit $B_{12}, 4800$ mg, ác. fólico, $1200 \mathrm{mg}$; pantotenato Ca $12000 \mathrm{mg}$; Vit. C, $48000 \mathrm{mg}$; biotina, $48 \mathrm{mg}$; cloreto de colina, $108000 \mathrm{mg}$; niacina, $24000 \mathrm{mg}$; e premix mineral comercial $(1 \mathrm{~kg} / \mathrm{t})$, com níveis de garantia por quilograma do produto: Fe, 50000 mg; Cu, 3000 mg; 20000 mg; Mn, 20000 mg; Zn, 3000 mg; I, 100 mg; Co, 10 mg; Se, 100 mg 
NAVARRO, FERREIRA, RIBEIRO FILHO, BOTION, PEREIRA, SILVAE MACIEL

formar uma granulometria homogênea. No início do experimento, cinqüenta peixes foram abtidos para posterior análise de carcaça. Depois do abate, as carcaças foram pesadas em balança de precisão $0,001 \mathrm{~g}$ com o objetivo de analisar e determinar a composição inicial da carcaça em teor de água, proteína e extrato etéreo.

Para determinação do glicogênio hepático e do músculo da linha dorsal, 10 peixes de cada tratamento dos estoques finais, mantidos em jejum, foram anestesiados e dissecados para retirada dos fígados e músculos. Esses tecidos foram colocados em microtubos $(1,5 \mathrm{ml})$, congelados imediatamente por imersão em nitrogênio líquido e armazenados em congelador $\left(-80^{\circ} \mathrm{C}\right)$ para posterior determinação da quantidade de glicogênio tecidual. Foram coletadas $0,5 \mathrm{~g}$ de cada tecido e colocados em um tubo contendo $\mathrm{KOH}$ a $30 \%$ para determinação da concentração de glicogênio, conforme o método da antrona descrito por Carrol et al. (1956).

As análises dos ingredientes utilizados nas dietas e das amostras dos peixes foram realizadas no Laboratório de Nutrição Animal do Departamento de Zootecnia (LNA/ DZO) da Universidade Federal de Minas Gerais - UFMG - conforme procedimentos descritos por AOAC (1995).

Foram analisados os parâmetros nutricionais de peso médio final ( $\mathrm{g})(\mathrm{PMF})$, ganho de peso (GP), comprimento total (cm) (CT), comprimento padrão $(\mathrm{CP})$, taxa de eficiência protéica (TEP), taxa de sobrevivência (TS).

Ganho de peso dos peixes (GP) foi calculado pela diferença dos pesos médios da parcela final e inicial:

$\mathrm{GP}=$ peso final $(\mathrm{g})$ - peso inicial $(\mathrm{g})$

Conversão alimentar aparente

$\mathrm{CAA}=$ alimento ingerido $(\mathrm{g}) / \mathrm{GP}(\mathrm{g})$

Taxa de eficiência protéica

$\mathrm{TEP}=\mathrm{GP}(\mathrm{g}) /$ proteína ingerida $(\mathrm{g})$

Foram analisados a composição química da carcaça (extrato etéreo e proteína), a porcentagem de proteína no ganho de peso (\%PBGP), a porcentagem de gordura no ganho de peso (\%GGP), o índice hepatossomático (IHS), o índice viscerossomático (IVS).

Porcentagem de proteína no ganho de peso

\%PBGP= Proteína corporal final $(\mathrm{g})$ - proteína corporal inicial (g)/ganho de peso $(g)$

Porcentagem de gordura no ganho de peso

\% GGP = gordura corporal final $(g)$ - gordura corporal inicial (g)/ganho de peso $(\mathrm{g})$

Índice viscerossomático (IVS) e indice hepatossomático (IHS),

IVS= PV/PC $\times 100$

IHS $=P F / P C \times 100$

$\mathrm{PC}=$ peso corporal

$\mathrm{PF}=$ peso figado

$\mathrm{PV}=$ peso da víscera

A taxa de crescimento específico foi determinada pela fórmula:

$\mathrm{TCE}=(\mathrm{In})$ peso total final $-(\mathrm{In})$ peso total inicial dividido pelo tempo de experimento

Os efeitos da suplementação de vitamina $\mathrm{C}$ foram submetidos à análise de variância ao nível de 5\% de significância pelo programa estatístico SAS (1997) e em caso de diferença os dados foram comparados pelo teste de separação de médias StudentNewman-Keuls.

\section{RESULTADOSEDISCUSSÃO}

Valores médios obtidos de temperatura foi de $28,23 \pm 0,63^{\circ} \mathrm{C} ; 7,25 \pm 0,58$ para $\mathrm{pH} ; 5,23$ $\pm 0,85 \mathrm{mg} \mathrm{l}^{-1}$ para oxigênio dissolvido, e permaneceram dentro da condições ótimas para o crescimento da espécie de acordo (Kubitza, 2000).

Observou-se efeito significativo $(\mathrm{p}<$ $0,05)$ no peso médio final, ganho de peso para tilápia revertida alimentada com 50,100 e $200 \mathrm{mg}$ de vitamina $\mathrm{C}$ por $\mathrm{kg}$ de ração (tabela II). Observou-se diferença significativa para conversão alimentar aparente, onde o tratamento com suplementação 50, 100,150 e $200 \mathrm{mg} / \mathrm{kg}$ de vitamina C obteve melhor resultado. Esses resultados sugerem que a vitamina $\mathrm{C}$ age na formação do colá- 


\section{DESEMPENHO DE TILÁPIA DO NILO COM VITAMINA C}

geno melhorando assim o desempenho zootécnico.

Segundo Rotta (2003), o ácido ascórbico influencia diretamente o crescimento dos peixes, pois tem função importante na formação do colágeno, que é o principal componente do esqueleto. Entretanto, outros estudos como o de Mello et al. (1999) não observaram diferenças significativas no ganho de peso e na taxa de sobrevivência de alevinos de piauçu para suplementação de vitamina C, de $50 \mathrm{mg}$ a $850 \mathrm{mg} / \mathrm{kg}$. Mesmo resultado foi relatado por Almeida (2003), que não observou diferenças $(p>0,05)$ no desempenho de pacu alimentado com suplementação de vitamina $\mathrm{C}$.

A suplementação de ácido ascórbico na dieta implica, também, no incremento de ganho de peso, de melhor taxa de conversão alimentar e de maiores taxas de sobrevivência, como relatado com Clarias gariepinus e Oreochromis niloticus x $O$. aureus (híbridos de tilápia) (Shiau e Hsu, 1999; Adham et al., 2000). Em alevinos de piauçu, a suplementação de vitamina $\mathrm{C}$, com doses entre 50 e $850 \mathrm{mg} / \mathrm{kg}$ de ração, não apresentou influência significativa no ganho de peso e na taxa de sobrevivência (Mello et al., 1999).

No estudo de Toyama (1999) com suplementação de vitamina $\mathrm{C}$ para tilápia na reversão sexual, encontrou-se melhor desempenho para valores de $859,5 \mathrm{mg}$ e $765,0 \mathrm{mg}$ de vitamina $\mathrm{C} / \mathrm{kg}$ da dieta, respectivamente para ganho de peso e de comprimento.

Não foi observada diferença significativa para o comprimento total. No entanto para o comprimento padrão foi observada diferença significativa com os tratamentos 50,100 e $200 \mathrm{mg} / \mathrm{kg}$ de vitamina C (tabela II). Já Wang et al. (2003), trabalhando com (Oplegnathus fasciatus), com seis níveis de vitamina C: $0,60,120,240,480$ e $2000 \mathrm{mg} \mathrm{L}$ ácido ascórbico (AA) por $\mathrm{kg}$ de dieta, observaram que o nível de $118 \mathrm{mg} / \mathrm{kg}$ de dieta obteve mais crescimento.

Verificou-se efeito significativo $(p<0,05)$ na taxa de eficiência protéica com suplementação de 50, 100, 150 e $200 \mathrm{mg} / \mathrm{kg}$ de vitamina $\mathrm{C}$, esse resultado pode ser explicado pela função da vitamina $\mathrm{C}$ de participar da síntese de colágeno. A taxa de sobrevivência foi estatisticamente significativo

Tabela II. Peso médio final (PMF), ganho de peso (GP), conversão alimentar aparente $(C A A)$, comprimento total (CT), comprimento padrão (CP), taxa de eficiência protéica (TEP) e taxa de sobrevivência (TS) de alevinos de tilápia alimentados com dietas com suplementação de vitamina $C$. (Final weight (PMF), weight gain (GP) daily weight gain (CAA), total lenght (CT), standard lenght (CP), protein efficiency rate (TEP) and survival rates (TS) of fingerlings of tilapia fed diets with supplementation of vitamin C).

\begin{tabular}{lcccccc}
\hline \multicolumn{5}{c}{ Vitamina C mg/kg } \\
& 0 & 50 & 100 & 150 & 200 & CV \\
\hline PMF (g) & $51,30 \pm 11,9^{\mathrm{b}}$ & $56,81 \pm 11,6^{\mathrm{a}}$ & $58,09 \pm 11,27^{\mathrm{a}}$ & $53,49 \pm 11,5^{\mathrm{ab}}$ & $57,5 \pm 11,2^{\mathrm{a}}$ & 20,7 \\
GP $(\mathrm{g})$ & $49,62 \pm 11,99^{\mathrm{b}}$ & $55,1 \pm 11,6^{\mathrm{a}}$ & $56,38 \pm 11,26^{\mathrm{a}}$ & $51,78 \pm 11,5^{\mathrm{ab}}$ & $55,8 \pm 11,2^{\mathrm{a}}$ & 21,4 \\
CAA & $1,70 \pm 0,52^{\mathrm{a}}$ & $1,52 \pm 0,52^{\mathrm{ab}}$ & $1,47 \pm 0,37^{\mathrm{b}}$ & $1,50 \pm 0,41^{\mathrm{b}}$ & $1,49 \pm 0,48^{\mathrm{b}}$ & 28,83 \\
CT $(\mathrm{cm})$ & $13,95 \pm 0,98$ & $14,36 \pm 1,0$ & $14,39 \pm 0,86$ & $14,2 \pm 1,0$ & $14,36 \pm 1,0$ & 6,98 \\
CP $(\mathrm{cm})$ & $10,41 \pm 0,82^{\mathrm{b}}$ & $10,82 \pm 0,76^{\mathrm{a}}$ & $10,84 \pm 0,67^{\mathrm{a}}$ & $10,65 \pm 0,79^{\mathrm{ab}}$ & $10,94 \pm 0,7^{\mathrm{a}}$ & 7,09 \\
TEP & $1,37 \pm 0,33^{\mathrm{b}}$ & $1,53 \pm 0,32^{\mathrm{a}}$ & $1,56 \pm 0,31^{\mathrm{a}}$ & $1,80 \pm 0,29^{\mathrm{ab}}$ & $1,55 \pm 0,3^{\mathrm{a}}$ & 29,2 \\
TS & $90,0 \pm 1,41^{\mathrm{b}}$ & $98,33 \pm 0,47^{\mathrm{a}}$ & $95,00 \pm 0,70^{\mathrm{b}}$ & $98,33 \pm 0,47^{\mathrm{a}}$ & $96,67 \pm 0,2^{\mathrm{b}}$ & 80,0 \\
\hline
\end{tabular}

Letras distintas na mesma linha indicam diferença significativa $(p<0,05)$ pelo teste de Student-NewmanKeuls. Valores em média \pm erro padrão. $C V=$ coeficiente de variação. 
Tabela III. Matéria seca (MS), proteína bruta (PB), extrato etéreo (EE), porcentagem de proteina no ganho de peso (PBGP), porcentagem de gordura no ganho de peso (GGP), índice viscerossomático (IVS) e indice hepatossomático (IHS) de alevinos de tilápia alimentados com dietas com suplementação de vitamina $C$. (Dry matter (MS), crude protein (PB), ether extract $(E E)$, weigth gain protein percentage (PBGP), weigth gain fat percentage (GGP), viscera somatic index (IVS) and liver somatic index (IHS) of fingerlings of tilapia fed diets with supplementation of vitamin C).

\begin{tabular}{lcccccc}
\hline \multicolumn{7}{c}{ Vitamina C mg/kg } \\
& 0 & 50 & 100 & 150 & 200 & CV \\
\hline MS\% & $26,18 \pm 0,28$ & $25,79 \pm 0,14$ & $25,57 \pm 0,49$ & $25,81 \pm 1,18$ & $25,92 \pm 3,21$ & 2,47 \\
PB\% & $57,02 \pm 1,0$ & $59,07 \pm 0,8$ & $56,77 \pm 1,6$ & $55,96 \pm 2,4$ & $56,26 \pm 1,4$ & 2,69 \\
EE\% & $20,75 \pm 2,0$ & $21,06 \pm 4,2$ & $20,01 \pm 2,71$ & $19,47 \pm 0,86$ & $19,17 \pm 1,82$ & 12,57 \\
PBGP\% & $55,73 \pm 0,9$ & $57,92 \pm 0,77$ & $55,59 \pm 1,6$ & $54,72 \pm 2,3$ & $55,12 \pm 1,4$ & 2,69 \\
GGP\% & $19,47 \pm 2,0$ & $19,91 \pm 4,28$ & $18,89 \pm 2,71$ & $18,24 \pm 0,86$ & $18,04 \pm 1,82$ & 12,57 \\
IVS & $10,34 \pm 1,66$ & $10,70 \pm 0,99$ & $10,30 \pm 2,02$ & $10,34 \pm 1,31$ & $10,57 \pm 1,79$ & 20,80 \\
IHS & $1,68 \pm 0,72$ & $1,65 \pm 0,73$ & $1,70 \pm 0,78$ & $1,46 \pm 0,69$ & $1,51 \pm 1,45$ & 50,44 \\
& & & & & & \\
\hline
\end{tabular}

Letras distintas na mesma linha indicam diferença significativa $(p<0,05)$ pelo teste de Student-NewmanKeuls. Valores em média \pm erro padrão. $C V=$ coeficiente de variação.

para os tratamentos com suplementação de 50 e $150 \mathrm{mg}$ de vitamina $\mathrm{C} / \mathrm{kg}$. Outros autores observaram efeito positivo na influencia da vitamina $C$ na taxa de sobrevivência Mello et al. (1999) e Rotta, (2003). Não foi observada diferença significativa para índice viscerossomático e índice hepatossomático (tabela III).

A suplementação de vitamina $C$ não influenciou $(p>0,05)$ a porcentagem de MS, proteína bruta e porcentagem de proteína no ganho de peso (tabela III). Não foi observada diferença significativa $(p>0,05)$ dos tratamentos para extrato etéreo, porcen- tagem de gordura no ganho de peso, no entanto a porcentagem de extrato etéreo diminuiu com aumento da suplementação de vitamina $\mathrm{C}$, provavelmente devido à função da vitamina $\mathrm{C}$ de participar da biossíntese de carnitina como relatado por Rotta (2003).

A suplementação de vitamina $C$ não influenciou significativamente $(p<0,05)$ a concentração de glicogênio no músculo (tabela IV). Não foi observada diferença significativa $(\mathrm{p}>0,05)$ para glicogênio do fígado (tabela IV). Henrique et al. (1998) relataram que a suplementação de vitamina

Tabela IV. Concentração de glicogênio do fígado (\%) e concentração de glicogênio do músculo (\%) de alevinos de tilápia alimentados com dietas com suplementação de vitamina $\mathrm{C}$ na ração. (Concentration of glycogen in the liver (\%) and the concentration of muscle glycogen (\%) from the tilapia fingerlings fed diets with supplementation of vitamin $\mathrm{C}$ ).

\begin{tabular}{lcccccc}
\hline \multicolumn{7}{c}{ Vitamina C $(\mathrm{mg} / \mathrm{kg})$} \\
& 0 & 50 & 100 & 150 & 200 & CV \\
\hline Fígado & $8,25 \pm 0,05$ & $7,11 \pm 0,08$ & $8,31 \pm 0,07$ & $5,04 \pm 0,03$ & $5,70 \pm 0,04$ & 14,06 \\
Músculo & $0,048 \pm 0,02$ & $0,11 \pm 0,01$ & $0,13 \pm 0,01$ & $0,05 \pm 0,03$ & $0,10 \pm 0,01$ & 4,39 \\
\hline
\end{tabular}

Letras distintas na mesma linha indicam diferença significativa $(p<0,05)$ pelo teste de Student-NewmanKeuls. Valores em média \pm erro padrão. $C V=$ coeficiente de variação. 


\section{DESEMPENHO DE TILÁPIA DO NILO COM VITAMINAC}

C em peixes reduz a glicemia em situações de stress.

Alterações da concentração de glicogênio têm sido relacionadas em diferentes tecidos com o modo de vida do animal, estágio de desenvolvimento gonadal, estação do ano e sexo (Oliveira et al., 1997). A maior quantidade no músculo vermelho, quando comparado ao músculo branco, parece ser resultante da atividade de cada um deles; o músculo vermelho responde pelo esforço da natação em condições normais perdendo menos energia, enquanto

\section{BIBLIOGRAFIA}

Adham, K.G., Hashem, H.O., Abu-Shabana, M.B. and Kamel, A.H. 2000. Vitamin C deficiency in the catfish Clarias gariepinus. Aquacult. Nutr., 6: 129-139.

Almeida, G.S.C. 2003. Suplementação dietética de vitamina $\mathrm{C}$, desenvolvimento e sanidade do Pacu (Piaractus mesopotamicus Holmberg, 1887). (Dissertação de mestrado). Escola Superior de Agricultura Luis de Queiroz. Piracicaba. $47 \mathrm{pp}$.

AOAC. 1995. Official Methods of Analysis of AOAC International. P. Cunniff (ed.). $16^{\text {th }}$ ed. Arlington. vol. I e II. 469 pp.

Carrol, N.V., Longley, W. and Roe, J.H. 1956. The determination of glycogen in liver and muscle by use of anthrone reagent. J. Biol. Chem., 220: 583-593.

Cyrino, J.E.P., Portz, L. e Martino, Y.R. 2000. Retenção de proteína e energia em juvenis de black bass Micropterus salmoides. Sci. Agricola, 57: 609-616.

Henrique, M.M.F., Gomes, A.E.F. and GouillouCoustans, M.F. 1998. Influence of supplementation of practical diets with vitamin $\mathrm{C}$ on growth and response to hypoxic stress of seabream, Sparus aurata. Aquaculture, 161: 415-426.

Hepher, B. 1988. Nutrition of pond fisher. Cambridge University. Cambridge. 387 pp.

Kubitza, F. 2000. Tilápia tecnologia e planejamento na produção comercial, $1^{\mathrm{a}}$ ed. 289 pp.

Lee, K-J. and Bai, S.C. 1998. Different dietary levels of L-ascorbic acid affect growth and vitamin $\mathrm{E}$ status of juvenile Korean rockfish, o músculo branco responsável pela atividade mais vigorosa, tais como captura de presas apresenta maior desgaste energético (Hepher, 1988, Oliveira et al., 1997).

\section{CONCLUSÕES}

A suplementação de 50,100, 150 e 200 $\mathrm{mg} / \mathrm{kg}$ de vitamina $\mathrm{C}$ apresentou desempenho produtivo satisfatório em tilápias revertidas. Para utilizar dietas com menor custo é recomendável o uso de $50 \mathrm{mg} / \mathrm{kg}$ de vitamina $\mathrm{C}$.

Sebastes schlegeli. Aquaculture, 161: 475-477. Matty, A.J. 1985. Nutricion and aquaculture, Outlook on Agriculture, 14: 14-20.

Mello, R.F., Moura, M.A.M., Vieira, I. e Cyrino, J.E.P. 1999. Suplementação da dieta de alevinos de piauçu (Leporinus obtusidens) com vitamina C. Sci. Agricola, 56: 1223-1231.

Merchie, G., Lavens, P., Verreth, J., Ollevier, F., Nelis, H., De Leenheer, A., Storch, V. and Sorgeloos, P. 1997. The effect of suplemental ascorbic acid in inriched live food for Clarias garienpnus larvae at startfeeding. Aquaculture, 151: 245-258.

NRC. 1993. Requirement of Fish $2^{\text {nd }}$ ed. National Academy Press. Washington D.C. 114 pp.

Navarro, R.D., Silva, R.F, Ribeiro Filho, O.P., Calado, L.L., Rezende, F.P., Silva, C.S. e Santos L.C. 2006. Comparação morfometrica e índices somáticos de machos e fêmeas do lambari prata (Astayanax scabripinnis Jerenyns, 1842) em diferente sistema de cultivo. Zootec. Tropical, 24: 165-176.

Navarro, R.D., Lanna, E.A.T., Donzele, J.L., Matta, S.L.P e Souza, M.A. 2007. Níveis de energia digestível da dieta sobre o desempenho de piauçu (Leporinus macrocephalus) em fase pós-larval. Acta Sci. Anim. Sci., 29: 109-114.

Oliveira, E.G., Urbinati, E.C., Souza, V.L. e Roviero, D.P. 1997. Concentrações de glicogênio em diferentes tecidos de pacu (Piaractus mesopotamicus, H. 1887). Boletim do Instituto de Pesca, 24: 89-95.

Rostagno, H.S. 2000. Composição de alimentos e exigência nutricional de aves e suínos (Tabela 
NAVARRO, FERREIRA, RIBEIRO FILHO, BOTION, PEREIRA, SILVAE MACIEL

Brasileira). UFV. Viçosa-MG. Impr. Univ. 141 pp.

Rotta, M.A. 2003. Utilização do ácido ascórbico (vitamina C) pelos peixes. Embrapa Pantanal. Corumbá. 54 pp.

SAS. 1997. Statistical Analysis System. SAS/ STAT user's guide. Version 6. 4. ed. Washington, DC. USA. 943 pp.

Shiau, S.Y. and Hsu, T.S. 1999. Quantification of vitamin $\mathrm{C}$ requirement for juvenile hybrid tilapia, Oreochromis niloticus $\mathrm{x}$ Oreochromis aureus, with L-ascorbyl-2- monophosphate- na and Lascorbyl-2-monophosphate-Mg. Aquaculture,
175: 317-326.

Toyama, G.N. 1999. Suplementação de vitamina C na reversão sexual de tilápia do nilo (Oreochromis miloticus). Anais do III Simpósio sobre Manejo e Nutrição de Peixes, Anais... Campinas. pp. 101-110.

Wang, X., Kang-Woong, K., Sungchul, C., Bai MinDo, H. and Byong-Youl, C. 2003. Effects of the different levels of dietary vitamin $C$ on growth and tissue ascorbic acid changes in parrot fish (Oplegnathus fasciatus). Aquaculture, 215: 203-211. 